\title{
Disposer Reposer Proposer
}

Entretien avec Pierre Giner

Julien Collonges et Pierre Giner

\section{(2) OpenEdition}

\section{Journals}

Édition électronique

URL : https://journals.openedition.org/rbnu/3484

DOI : $10.4000 /$ rbnu.3484

ISSN : 2679-6104

Éditeur

Bibliothèque nationale et universitaire de Strasbourg

\section{Édition imprimée}

Date de publication : 1 mai 2011

Pagination : 68-79

ISSN : 2109-2761

Référence électronique

Julien Collonges et Pierre Giner, « Disposer Reposer Proposer », La Revue de la BNU [En ligne], 3 | 2011, mis en ligne le 01 mai 2011, consulté le 08 août 2021. URL : http://journals.openedition.org/rbnu/3484 ; DOI : https://doi.org/10.4000/rbnu.3484

\section{c) (i) (2)}

La Revue de la BNU est mise à disposition selon les termes de la Licence Creative Commons Attribution - Pas d'Utilisation Commerciale - Partage dans les Mêmes Conditions 4.0 International. 


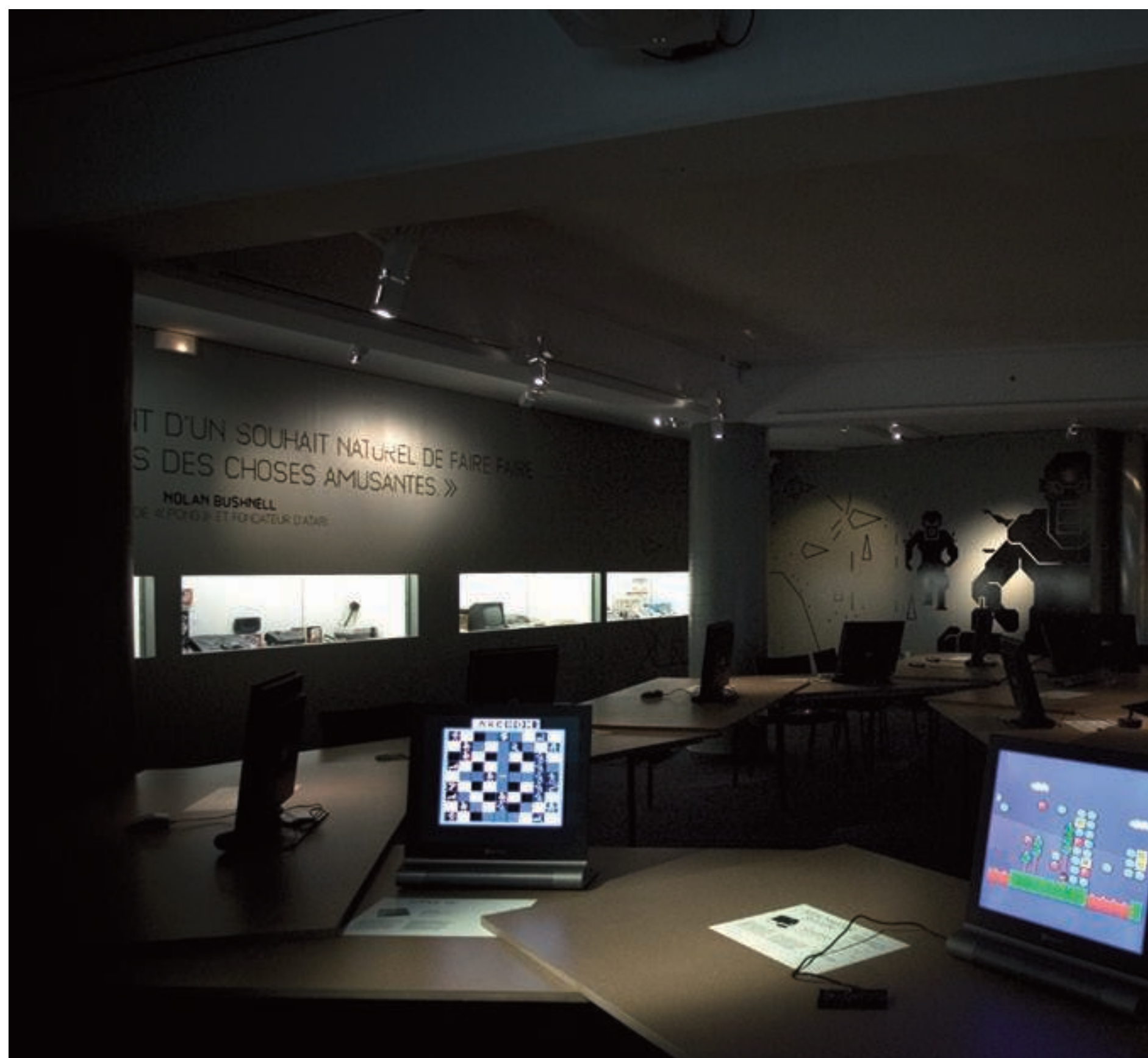

Les visiteurs sont invités à tester les jeux qui ont marqué les trente premières années de l'histoire des jeux vidéo (exposition Museogames au Musée EDF Electropolis à Mulhouse) 


\section{DISPOSER REPOSER PROPOSER}

Entretien avec Pierre Giner

Artiste contemporain, Pierre Giner a travaillé sur de nombreuses expositions ${ }^{1}$, dont la dernière, Museogames, consacrée aux jeux vidéo, a rencontré un vif succès au musée du CNAM à Paris, et est actuellement montrée au Musée EDF Electropolis de Mulhouse'. Il développe ici quelques-unes de ses idées sur l'art et la manière de monter une exposition. 


\section{Pierre Giner, vous considérez-vous comme un scénographe?}

Pas vraiment. Je n'ai pas d'écriture particulière des objets, pas de "style ". C'est un métier, avec ses codes, que je connais peu et ne souhaite pas reproduire. Je préfère produire une scène que mettre en scène. Trouver quelles formes produisent des ensembles : des lieux, des gens, leurs activités, leurs pratiques, des ensembles de documents. Ces organismes produisent des formes et je m'efforce de trouver le dispositif le mieux adapté à la question qui m'est posée.

\section{Dans votre travail, ce dispositif inclut des éléments qu'habituellement on n'expose pas, appartenant davantage aux coulisses d'une exposition classique ou relatifs à l'institution qui abrite l'exposition.}

Oui, car il faut montrer l'institution si l'on veut faire comprendre la complexité de ce qui est exposé. Il s'agit de rendre disponible cette complexité, mais aussi de prendre plaisir à la voir et d'y participer. C'est donc un dispositif d'accueil, de participation, mais aussi de production d'un objet visuel, d'une scène, intelligente et sensible, où l'on participe du sujet. Non pas au sens de l'interactivité, mais au sens d'être au présent avec le sujet. J'aime l'étymologie d'intéresser, inter-esse, être pris au sein de. C'est le point de départ de tout, une immersion du corps et du sujet qui s'oppose à l'idée d'un rapport frontal du visiteur à l'objet. Pour Museogames, l'idée était de produire une grande image qui soit « jouable » et dans laquelle on puisse s'intégrer. Une " mise en vue " immédiate de l'ensemble, incluant aussi une chronologie et toute une série d'éléments. Ensuite, je dispose ces éléments, je leur trouve une place, un usage. Mais plutôt que de calquer une histoire sur des objets, dans une démarche d'illustration, j'essaie de créer une sorte de générateur autonome qui développerait cette histoire par lui-même, grâce aux relations qui s'établissent entre les objets. Comme avec le "générateur de collections " misc_spaces, un logiciel qui, dans l'exposition de la FRAC ChampagneArdennes en 2004, permettait au spectateur de disposer virtuellement à sa guise les œuvres dans l'espace de l'exposition.

\section{C'est alors le visiteur qui est mis à contribution et devient acteur et producteur de l'exposition...}

Oui, je me méfie de l'idée de médiation. Je préfère l'" immédiation ". Je n'aime pas que l'on ne fasse pas confiance au spectateur, terme que je préfère à celui de visiteur parce que je construis les choses de manière visuelle. Museogames est ainsi une grande scène autour de laquelle gravitent quelques points de vue possibles du spectateur. La démarche est simple. Je résous les choses visuellement en me demandant " que voit-on ?", " comment est-ce que ça prend sens ? ", à partir de configurations élémentaires : être pris dans la scène en train de jouer, jouer devant un écran, regarder celui qui joue, regarder ce qui est joué comme des tableaux, s'isoler pour se consacrer à un sujet, passer de la réserve à la salle de jeux et éprouver un contraste entre un objet domestique et public, etc. Le dispositif est construit comme un montage cinématographique : on passe d'un plan à l'autre et ce qui importe, c'est l'articulation des plans, les contrastes. L'idée est de donner en une séquence l'ensemble des constituants fondamentaux qui rendent compte de la complexité du sujet (stock, processus de sélection, usages, discours). Mais sans mêler ces éléments. Car l'installation est devenue une forme artistique consacrée : le spectateur sait lire des installations, produire du sens en circulant d'un objet à l'autre. Nous n'avons donc pas à les relier pour lui : il produit lui-même le contenu de l'exposition. Bien sûr, il s'implique plus ou moins longtemps. Mais il faut lui donner des outils, lui proposer des médias divers. Je ne veux pas forcer l'autre : juste lui fournir ce dont il a besoin. Pour Museogames, nous prévoyons aussi d'éditer un catalogue qui fixera les contenus d'une autre manière encore. On propose ainsi des médias croisés, une superposition de couches qui se complètent : les jeux, l'accueil, la compréhension, le catalogue, etc.

Dans Museogames il n'y a ni socles, ni vitrines. Ce refus des dispositifs de légitimation traduit une volonté de désacraliser l'exposition : traditionnellement, " ex-poser " un objet, c'est l'abstraire de sa banalité pour le transporter dans l'espace sacré du musée. Or votre démarche semble être inverse, comme s'il s'agissait de " re-poser " l'objet dans ses usages concrets et vivants.

Oui, il s'agit de retrouver l'objet et de clarifier sa position. J'aime ce mot " exposer ", y compris au simple 
sens de poser un objet. Au fond, mon travail, c'est cela et, du coup, je dois faire le tri. Pour misc_spaces, j’ai été confronté à ce problème. En montrant l'ensemble des collections à l'état de stock, puis en proposant un " générateur de collections ", on circulait entre deux absolus : d'un côté tout montrer - en se demandant : comment accéder à ce tout ? - et de l'autre côté, choisir en se demandant : selon quels critères ? Or j'aime les musées parce qu'ils accomplissent pour moi ce travail capital de constitution des fonds : ils collectionnent et conservent des objets et nous permettent de voyager dans ces fonds comme dans notre propre mémoire. J'aime aussi que d'autres que moi fassent un choix et me proposent une lecture à travers leur sélection. Ensuite, c'est le dialogue entre cette mémoire stockée et la parole qu'elle produit - fût-ce par le seul choix des objets qui m’intéresse. Ce qui me dérange dans le rôle du commissaire d'exposition, c'est que s'il peut proposer une lecture de l'objet exposé, il n'est pas le seul : c'est aussi le cas des sociologues, des producteurs ou des acteurs de tel domaine, et, finalement, de n'importe qui. Or au fond, l'enjeu de ces pratiques - collectionner, exposer - c'est de produire de l'intelligence et de la parole : ça nous sert à nous dire et à dire des choses. Du coup, si j'essaie de montrer le fonctionnement de l'objet, je ne veux pas en fixer complètement la lecture. Seulement permettre d'en faire des lectures. Après tout, l'expert et l'amateur ont les mêmes pratiques. L'expert a juste accès à des outils privilégiés. Mais le spectateur contemporain est aussi très instruit. Il vit au milieu de la culture, il y a accès quand il le veut, surtout dans nos pays où la culture est publique. Il a donc la même pratique que l'expert, mais une pratique pour lui-même. L'enjeu est alors le dialogue entre des paroles diverses et toutes aussi légitimes.

C'est encore plus manifeste avec le jeu vidéo, qui n'est pas encore sacralisé comme d'autres médias. Or c'est lorsqu'un objet culturel est consacré que la parole de l'expert devient dominante et éclipse les autres lectures en imposant une problématique de médiation. L'intérêt du jeu vidéo est-il d'être encore " profane " ?

Oui. Cela n'invalide pas la médiation, mais mon rôle est d'abord de permettre le spectre le plus large possible des discours sur l'objet. Une exposition commence quand on se demande « ça veut dire quoi ? » ou « qu'est- ce que ça me dit, à moi ? " Je profite alors de cette perplexité pour fournir au spectateur des éléments pour enrichir cette question et des outils pour collecter des réponses. En ce sens, une exposition est un objet très démocratique. Une société est démocratique dès lors qu'elle cherche en permanence à se donner une forme démocratique. Et une exposition participe de cette recherche.

Il y a dans votre démarche un désir de remettre les objets en situation. En même temps, le regard artistique se caractérise souvent par la mise entre parenthèses des relations quotidiennes avec les objets. Vous-même avez recours à ces déplacements qui font apparaître les objets sous un jour nouveau (ainsi, dans Museogames, ces écrans projetant des débuts de jeux accrochés comme des tableaux). Comment conciliez-vous ces deux démarches?

C'est lié à une conception du collectif : je travaille sur des objets privés que je rends publics. Le jeu vidéo est une pratique solitaire, mais ce qui me plaît, c'est de voir jouer l'autre, de faire apparaître des déplacements entre privé et public. Je ne sais d'ailleurs pas ce qu'est "le " public. Nos sociétés modernes sont construites sur des agrégats d'individus dotés de la capacité de se regrouper. Je pars donc d'une situation de dispersion avec le désir de produire un lieu d'échange et de réunion. Pour Museogames, je me suis inspiré des salles de jeux de Tokyo, où l'on peut aussi bien jouer que consulter ses e-mails, lire des mangas ou même dormir. C'est juste de l'accueil organisé autour de contenus qui relèvent à la fois de la culture et de la consommation. Aujourd'hui la consommation est si complexe, intègre tellement de choix, qu'il est difficile de la dissocier de la culture. Certes, on la subit en partie, mais c'est souvent par la consommation que l'on accède au culturel : de la bibliothèque au centre commercial, les chemins qui mènent à la culture sont de plus en plus sophistiqués. En ce sens, la salle de jeux est un lieu de vie qui correspond à un certain usage de la culture.

Le musée, lui, est plus inactuel. En tout cas, ses formes n'ont pas été actualisées. La façon actuelle d'exposer des tableaux dans de grandes pièces blanches - le " white cube » - est née à la galerie Durand-Ruel qui exposait les impressionnistes au XIX ${ }^{e}$ siècle. C'est une forme 


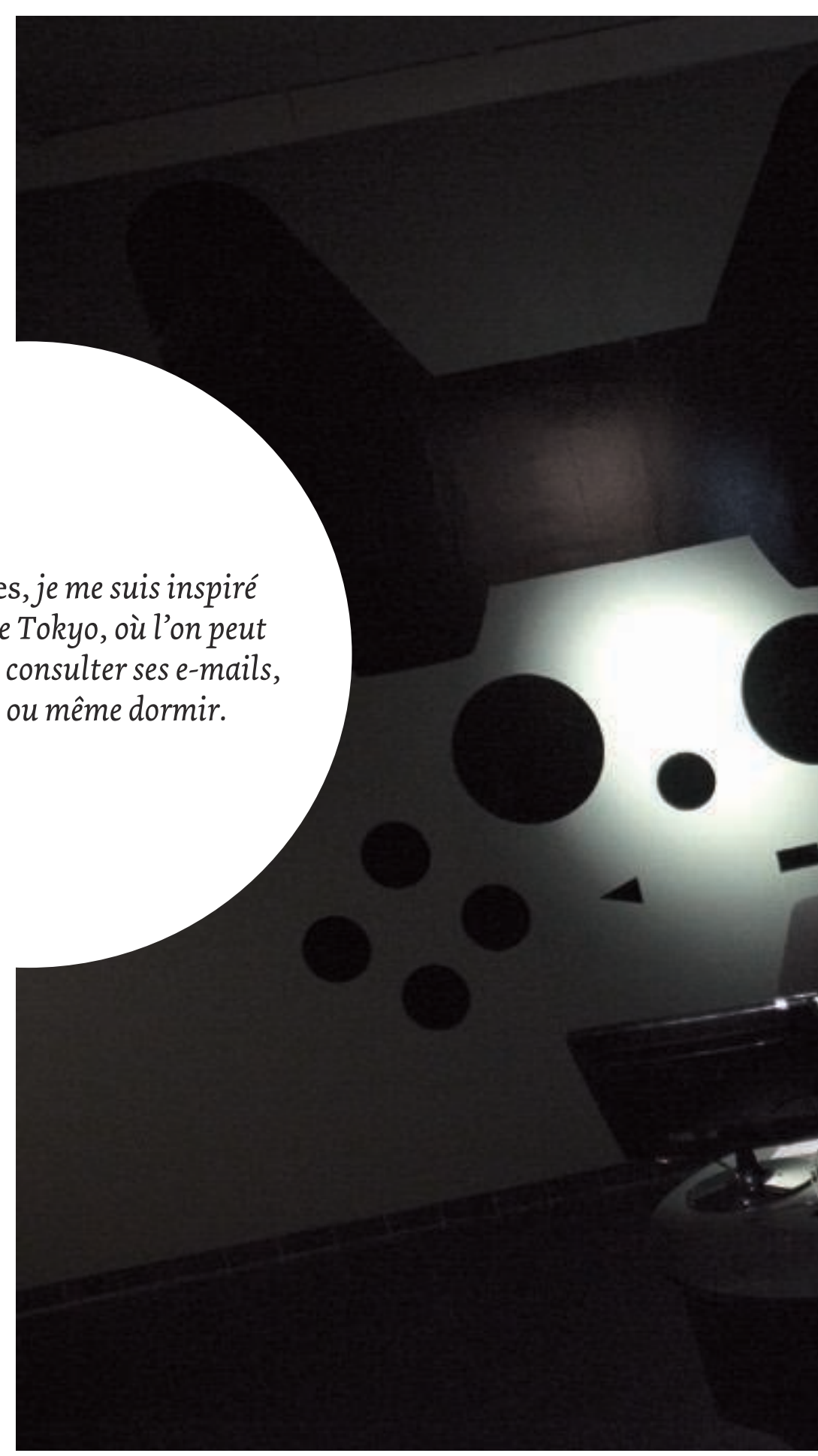

Pour Museogames, je me suis inspiré des salles de jeux de Tokyo, où l'on peut aussi bien jouer que consulter ses e-mails, lire des mangas ou même dormir. 


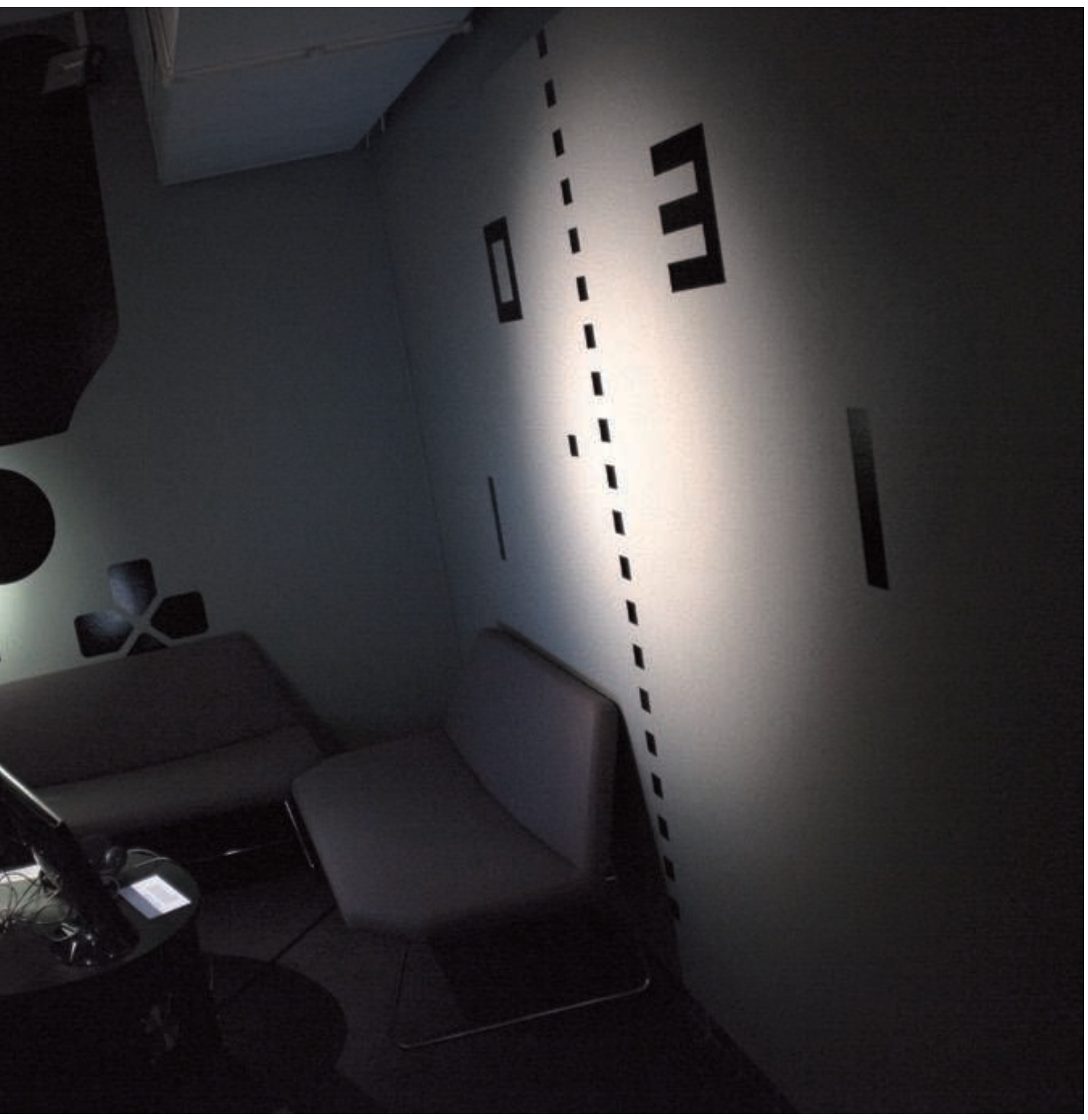

Exposition Museogames au Musée EDF Electropolis à Mulhouse 
que l'on croit actuelle, mais qui est liée au moment particulier de l'émancipation du tableau par rapport à ses conditions de production, le moment de la vente par lequel le tableau acquiert une vie autonome. Donc une forme liée à une modernité du siècle dernier. Or depuis, de nouveaux objets et de nouveaux usages sont apparus. Ce ne sont pas les tableaux qui sont dépassés, plutôt leurs modalités d'appropriation, et notamment les lieux. Certains musées ont figé les modalités d'appréhension des œuvres. Le " white cube " est ainsi devenu une forme un peu vide : c'est le " hub " mondial, partout le même objet, le même format. Partout identique et donc aussi nulle part. Au départ, Museogames devait aussi être une exposition toute blanche, avec socles et vitrines. Or la console n'est pas un objet artistique. D'abord parce qu'elle n'est qu'un terminal, le véritable objet étant le jeu luimême. Ensuite parce que l'intérêt est de considérer à la fois le jeu, la console, les usages. Au fond, ce qui m'intéresse, ce sont les objets augmentés. Et puis, il y a un formalisme de la sélection des objets, lié à des pratiques corporatistes. N'étant pas commissaire, je ne sélectionne donc pas les objets à partir de savoirs et de valeurs déjà constitués. Mais ça m’intéresse que d'autres le fassent.

\section{Mais si vous n'intervenez pas dans la sélection, comment pouvez-vous espérer faire bouger les pratiques, assouplir le formalisme de ces critères de sélection?}

Ce sont des pratiques très réglées. Et je m’intéresse plus à ceux qui écrivent des histoires avec ces objets, comme Olivier Saillard, du musée de la mode de la Ville de Paris, pour $i$-dance, ou Stéphane Natkin ${ }^{3}$ pour Museogames. N'émanant pas de ces domaines, je ne pense pas avoir la légitimité pour prendre la parole à leur sujet. Je préfere produire des lieux capables d'accueillir leur parole. C'est pourquoi mes expositions sont conçues comme des générateurs : pour que ces lieux soient vivants et m'informent, ils doivent être à la fois des lieux d'accueil et des lieux productifs - qui produisent ce dont ils sont le lieu, qui accueillent et produisent une parole sur l'objet exposé. Je travaille donc en groupe, en invitant des personnes capables de remplir les lieux, plutôt qu'en cherchant à épuiser un sujet. Je m'en sens incapable et surtout, ça n'est pas intéressant pour le spectateur. De ce point de vue, certaines expositions se ressentent encore trop de l'influence de l'école. Et on peut aussi poser la question de la valeur des objets exposés : pourquoi celui-ci en aurait-t-il plus que tel autre ? Mais ce qui m’intéresse surtout, c'est de distinguer des niveaux de contenus et des modalités d'accès, des usages, puis de scénariser ces différents niveaux.

Un autre aspect intéressant de votre travail est votre propension à inverser les rôles et à mettre en abyme l'exposition. Dans Museogames, le spectateur est à la fois le joueur, et donc l'acteur, de l'exposition, et celui que les autres peuvent regarder jouer, donc un des objets de l'exposition.

Surtout, pour moi, l'accueil est très important : il faut à la fois que les spectateurs soient accueillis, qu'on les voie et qu'ils participent de l'objet de l'exposition. Je ne tiens pas forcément à ce qu'on les regarde : ce n'est qu'une possibilité. Mais je ne vois pas pourquoi on les cacherait : ils font partie du sujet. Ce qui m'intéresse, c'est qu'on puisse le faire, qu'il y ait le moins de limitations possibles. Parce que je me méfie de ma propre intelligence, de mon propre manque d'imagination. Je ne veux pas que mon imagination limite l'objet. Au contraire, je cherche quelque chose qui puisse remettre en cause ce que j'imagine, remettre en marche mon imagination. C'est pourquoi je cherche des scénarios qui puissent accueillir des gens et susciter leur parole. Au fond, j'imagine les choses par rapport à des conversations susceptibles de stimuler mon imagination et, par là, de forger des objets. Ensuite, je me contente d' " augmenter » ces objets, de permettre à ces objets de se développer par eux-mêmes.

\section{Vous avez aussi coutume dans vos expositions d'en montrer les coulisses, par exemple en montrant les œuvres à l'état de stock. C'est une manière d'exposer les rouages de l'exposition, de permettre au spectateur de prendre conscience du lieu où il se trouve ?}

Oui, que l'on se pose la question : qu'est-ce que je fais là ? C'est cela l'actualité des pratiques culturelles, l'enjeu de l'art : l'œuvre n'est importante que si elle m'engage à dire quelque chose. On déplore souvent le désengagement propre à nos sociétés. Ce qui m'intéresse, c'est au contraire comment nous sommes tous engagés. Je cherche donc à produire un objet qui 
va vers le spectateur, lui propose un confort, le sollicite. Non pas sous la forme d'animations, mais plutôt en lui présentant un lieu où il puisse rester, un lieu de vie, de proposition, d'échange et, si possible, un lieu actuel. En me demandant : de quels lieux ai-je besoin ? En ce sens, le café, le lounge, la salle d'attente sont des lieux qui m'intéressent.

\section{Et la bibliothèque ?}

Bien sûr. Comme le musée. Ce sont des lieux qui me sont dédiés, et il est important qu'il me soient dédiés en particulier, ainsi qu'aux autres, en particulier. C'est aussi un lieu qui nous est dédié : d'abord à l'agrégat des individus, ensuite aux rencontres et regroupements des individus. On cherche trop souvent à s'adresser au " grand public ". C'est impossible. C'est une notion de marketing. L'objet culturel est adressé à l'individu avec, nécessairement, la possibilité de l'échec. Mais ce n'est pas grave. Un de mes amis, un peu perdu dans la société, a fait un voyage à New-York avec son école et, là-bas, a rencontré Malevitch : il s'est dit que Malevitch lui était adressé. Depuis, il est éditeur d'art. D'autres ne feront pas la même rencontre. Mais ils en feront d'autres. Bien sûr, c'est mieux si une exposition est fréquentée, mais elle n'est pas adressée à tout le monde. S'adresser à tous, c'est détruire le contenu. Il faut s'adresser à chacun, pas à tous.

Les lieux que vous évoquez sont hors des circuits culturels traditionnels. Ils possèdent leurs propres publics et usages. Comme s'il s'agissait plus de se greffer sur un lieu que d'y amener des publics...

Mais aujourd'hui, c'est vrai de tous les lieux. Du musée. De la bibliothèque : nous apprenons tous à lire, nous avons des livres, nous lisons sur nos téléphones portables. On a dépasséla question de l'accès général à la lecture. Après, savoir comment chacun, en particulier, peut y venir, ou comment traiter un sujet, sont des questions qui restent actuelles. Les bibliothèques sont des lieux passionnants. La BPI, par exemple : un lieu de vie et d'usages fabuleux, où l'on peut juste faire des photocopies ou se réchauffer au milieu des livres, mais aussi lire et mener une recherche. Je trouve bien que des gens se rencontrent grâce à la lecture, à un lieu, ou même seulement grâce à la table, ou au fait quel'endroit soit chauffé. C'est un objet très important dans notre société.

\section{Pour les bibliothèques, exposer le livre, c'est un peu la quadrature du cercle. Un livre se découvre d'abord en se lisant et une salle d'exposition semble souvent mal adaptée pour transmettre l'écrit. À tel point que l'on pourrait être tenté de renoncer à l'exposition au profit de cercles de lecture...}

L'enjeu, c'est de donner des livres à lire et de s'intéresser aux différentes modalités d'accès à la lecture. Pour ma part, je m'efforce de différencier les choses. Dans Museogames, par exemple, on montre des consoles qui ont été vendues à des millions d'exemplaires. Or qu'estce qui différencie la console du musée de celle que j'ai chez moi ? A priori, ce n'est pas un objet de valeur. Qu'est-ce donc qui lui donne de la valeur ? J'avais même proposé que l'on fabrique des fac-similés :j'étais intéressé par l'idée que les objets présentés n'aient pas d'original. Ou alors, qu'il faudrait aller chercher du côté de l'agence de design ou des ingénieurs ayant conçu la machine. Ensuite, comment regarde-t-on cet objet ? Regarde-t-on son design ? L'ouvre-t-on pour en voir la technologie ? Observe-t-on les usages ? Tous ces niveaux de compétence et de sens se superposent. Et en même temps, la question de l'original reste intéressante : où est l'original, l'âme, le radicant?

Cet original, ce peut être un mirage. Pour un livre, c'est aussi le contenu immatériel, reproductible à l'infini...

Oui, et ce caractère reproductible est intéressant. C'est une question très actuelle, celle de la diffusion numérique du livre - avec des acteurs comme Google qui oblige à se demander qui détient les droits de l'original. Et puis je trouve passionnants les gestes très instruits qu'implique le livre, en particulier dans la conservation. C'est pour cela que, dans Museogames, on montre des stocks. Et dans l'exposition du FRAC Champagne-Ardennes, les réserves : voir les caisses contenant les œuvres, avec les noms des artistes écrits dessus, ça me touche, c'est le début de quelque chose. Et cela permet de faire comprendre aux spectateurs que l'institution a d'autres œuvres et qu'elle a effectué un choix au sein du stock.

Le stock, en réalité, fait presque toujours partie du sujet d'une exposition. Il est un des niveaux qui, superposés, constituent l'objet exposé. L'original en est un autre. Or un original, un incunable par exemple, 

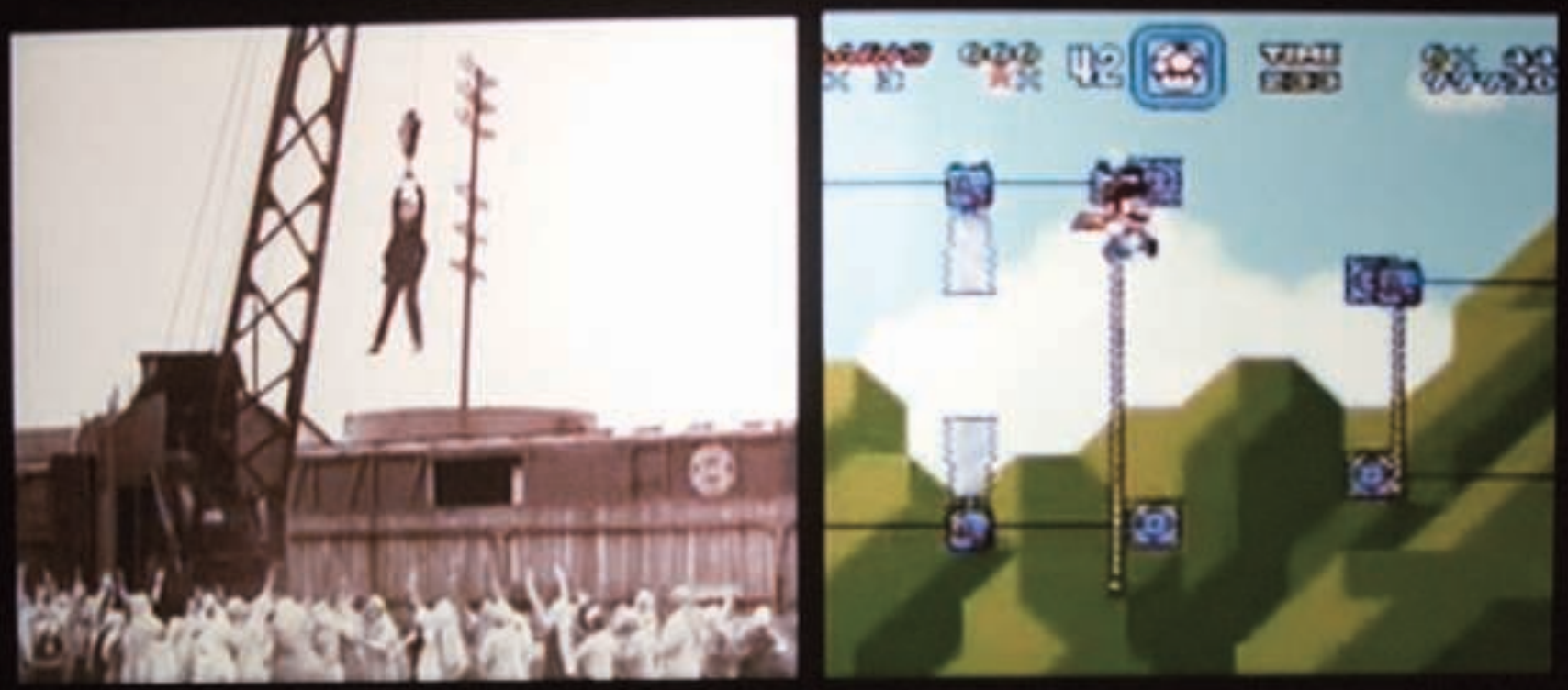

Le film Keaton vs. Mario de Jérôme Bosc confronte l'univers de Buster Keaton

(la fin de Seven chances, 1925) et des séquences du jeu Super Mario (1985).

Exposition Museogames au Musée EDF Electropolis à Mulhouse. 


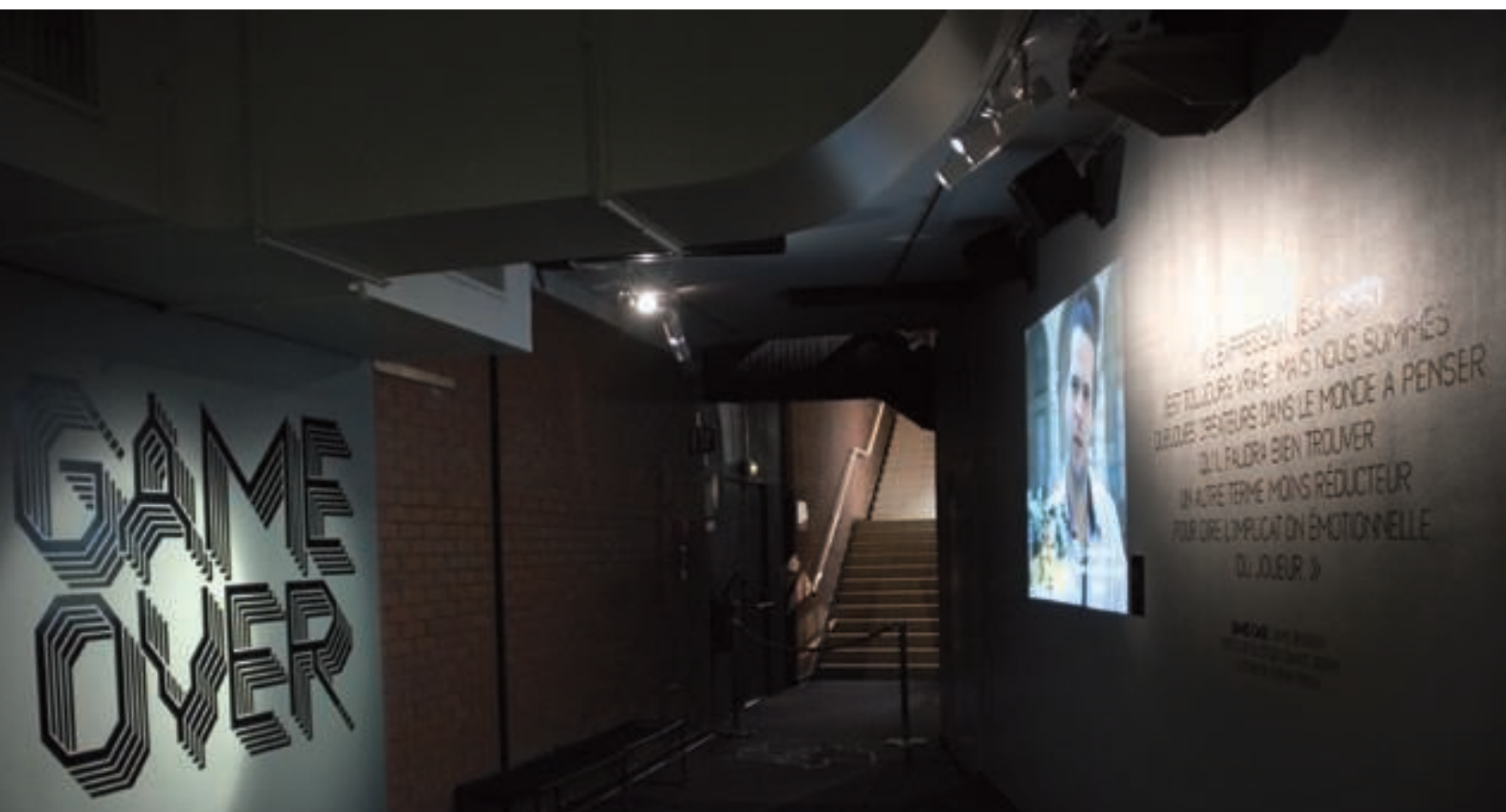

c'est certes très beau, mais on ne peut pas le toucher. Il a son propre mode de monstration, lié aux impératifs de conservation : chaque jour il est rangé dans sa boîte, entouré de feuilles de protection, puis on le sort pour le montrer, etc. Et seul le conservateur a la compétence et le geste juste. Mais si l'on veut passer de ce geste et de cet objet individuels à du collectif, il faut reproduire l'objet. Le filmer, le scanner. Produire ainsi un autre objet, consultable de façon publique, ce n'est pas trahir l'objet, c'est lui donner une fonction et un accès supplémentaires. Dans Misc_stories, le dispositif conçu pour l'exposition consacrée à l'architecte Patrick Bouchain à la biennale de Venise en 2006, il y avait une grande table, où l'on pouvait s'asseoir. Et sur cette table, des moniteurs, avec des images des projets de Patrick et un écran tactile permettant de feuilleter un livre qui était projeté sur le mur en très grand. Il s'agissait, grâceà la projection, de montrer les architectures quasiment à l'échelle. Et le choix du livre s'imposait parce que l'objet livre, même numérique, est le plus familier qui soit. Les tablettes numériques sont conçues et manipulées comme des livres et ces gestes et usages perdureront en dépit du passage du papier au numérique.

Ce qui est passionnant aujourd'hui, c'est qu'on peut faire des choses très diverses, augmenter l'objet d'origine en le déclinant ou en le reproduisant. Pour 
Museogames, je voulais montrer de l'édition, à partir d'un stock d'anciennes revues de jeux vidéos. Mais fallait-il montrer les originaux ? D'abord, ils sont fragiles. Ensuite, tout n'est pas intéressant dans tel ou tel numéro. Pour ma part, j’aurais préféré publier un nouveau numéro, plus intéressant et plus aisément consultable, à partir d'articles puisés dans le stock. Après tout, le livre est un objet de diffusion : il est normal de le rééditer. Or ce qui est extraordinaire dans les bibliothèques, c'est que, possédant de très nombreuses œuvres libres de droits, elles peuvent les dupliquer et les transmettre.

\section{Les nouvelles technologies n'ont-elles pas permis de prendre conscience que ce qui est au cœur de certaines œuvres, c'est avant tout quelque chose d'immatériel, indépendant des supports dans lesquels elles s'incarnent ?}

Jecrois plutôtqueces nouvellestechnologies définissent de nouvelles matérialités, qui s'ajoutent aux autres sans les supprimer. Je travaille actuellement pour le Fonds national d'art contemporain, qui m'a demandé de rendre public un fonds de plus de 40 ooo œuvres, en utilisant aussi bien le numérique que l'édition papier, les installations, la téléphonie mobile, etc. Ce faisant, on montre les œuvres, mais aussi la documentation des œuvres, c'est-à-dire à la fois leurs répliques sur d'autres supports et les commentaires qu'elles suscitent. Mais il faut veiller à ne pas oublier l'objet d'origine, qui n'est jamais remplacé. Ces avatars se placent à côté, forment une autre strate de sa complexité.

\section{Pour en revenir aux bibliothèques, si vous aviez à travailler sur une exposition autour du livre, comment vous y prendriez-vous?}

En partant du livre et de la pratique de la lecture. En m'inspirant par exemple du Musée du manga à Kyoto, un lieu où l'on peut s'installer et lire tous les mangas conservés dans le musée. Il y a aussi des expositions, qui sont comme des relectures de ce qui est conservé au musée, et donc du musée lui-même. C'est donc un espace qui produit sa propre compréhension de luimême et dans lequel le geste le plus important est celui de lire. L'important est d'être là, au milieu des livres, sachant que tous peuvent être lus. Ensuite, il y a quelques objets d'accueil très simples : des banquettes, des tables. Et les gens habitent vraiment là, ils achètent leur billet pour la journée.

\section{On pourrait alors imaginer une exposition se} contentant d'installer dans un lieu une sélection de livres sur un thème et d'inviter à la lecture... Oui, l'exposition serait une simple extraction d'une partie des fonds, et la table, le moyen le plus simple d'" exposer » les livres, au sens où exposer un livre, c'est d'abord le lire. Il n'est pas nécessaire que ce livre soit une édition rare : il suffit d'en proposer une reproduction, une édition tardive ou réalisée pour l'occasion. Après tout, les bibliothèques mettent à disposition des livres qui sont des reproductions précisément pour ne pas abîmer les originaux. Pourquoi ne pas réitérer ce geste pour une exposition? Ce qui n'empêche pas d'exposer l'original selon une autre modalité. Il s'agit moins de s'émanciper de l'original que de prendre un peu de distance avec l'idée d'objet unique et sacré. Sans pour autant l'évacuer. J'aime beaucoup le cérémonial avec lequel on montre les photographies dans les galeries, les gants que l'on porte pour ne pas les abîmer. Le rapport à l'original reste fascinant. Mais voir la même photo reproduite, déplacée, l'avoir pour soi, sous forme de carte postale, sont des usages et des appréhensions de l'objet différents, et porteurs de sens. Pour l'édition originale, on pourrait imaginer qu'au lieu d'être exposée en permanence dans une vitrine, elle soit sortie à certaines dates et certaines heures, et montrée au public en respectant et en exposant les procédures de conservation. Tous les niveaux sont intéressants.

\section{Un des effets de la numérisation a été d'effacer les frontières entre les manières d'exposer la culture. Les différences s'estompent entre revues et galeries en ligne, expositions virtuelles, blogs, bibliothèques numériques. Est-ce une vraie nouveauté ou bien le numérique n'est-il désormais qu'une forme parmi d'autres?}

Pour moi, les mosaïques de Constantinople valent les fresques de Florence, qui valent les tableaux de Chardin. Aucun n'a effacél'autre. Mais chaque nouveau média développe de nouveaux contenus et usages, en intégrant les anciens médias à ses propres contenus, en les augmentant, en les déplaçant. La question de la disparition du papier n'est donc pas pertinente. C'est un média tellement puissant, accessible, rapide, complet... Beaucoup plus que le numérique. Mais le numérique permet autre chose. Pour Museogames, j'aimerais proposer aussi un catalogue téléchargeable sur tablettes numériques et permettre ainsi d'autres 
objets de lecture, intégrant du texte, des images, des vidéos, et bien sûr les jeux de l'exposition. Pour que l'on puisse jouer ou rejouer l'exposition à distance.

\section{Autre chose, pour finir : pouvez-vous nous en dire plus sur le projet i-dance?}

C'est une installation. L'intérêt est que c'est un objet de contenu - un jeu vidéo pour apprendre à danser mais aussi un objet très esthétique. Au départ, on m'a demandé d'imaginer un objet pour les vingt ans $\mathrm{du}$ festival de mode d'Hyères. Je suis étranger au domaine, mais j'aime la légèreté du sujet, la façon dont les stylistes racontent des choses avec des corps et des vêtements. Et je trouve l'objet défilé très intéressant, commercial et créatif, à mi-chemin entre exposition et danse. Mon idée a été de coupler les modèles dessinés par les stylistes à une technologie qui fait danser des formes grâce à de la capture de mouvements. On peut alors faire danser les personnages, modifier leur apparence. C'est un générateur qui produit presque automatiquement des images autour de la danse, soit pré-articulées à la musique, soit projetées en live. Ensuite, je requalifie l'objet en termes de contenus : les différents contenus - danse, vêtements, personnages, musique - qui sont d'abord un objet privé, un objet d'écran, sont mis en place dans des lieux publics, parfois très spectaculaires, parfois plus modestes. Ensuite, l'objet se développe : j’ai invité le danseur et chorégraphe Christian Rizzo à produire de la capture de mouvements, j'invite régulièrement des DJs pour mettre de la musique sur cet objet. Au fond, j'aime que des gens dansent au sein de cet objet et moi, je me contente de faire de la direction artistique - agréger des contenus - et de la direction technologique - réinventer un objet à partir de la capture de mouvements. Et j'aime cette intégration de nouveaux contenus par le truchement de la danse. Parce que c'est inépuisable. Je vais prochainement travailler de nouveau avec des danseurs : on pourra alors à la fois danser, voir danser le danseur équipé du costume de capture de mouvements, voir son avatar danser, modifier cet avatar, voir cet avatar danser avec des danseurs déjà enregistrés, etc.

\section{Toujours cette confusion des rôles entre spectateur et joueur, danseur et spectateur...}

Cela correspond à ma position en tant qu'artiste : je suis moi-même dedans-dehors, comme nous tous. On a suffisamment de compréhension de l'art, des médias, de la culture, pour adopter cette double position. C'est pourquoi je me méfie de l'idée de médiation. Ce n'est pas grave de ne pas comprendre, de simplement vivre l'événement. Ce sont des questions que l'on emporte avec soi, y compris l'incompréhension. Comme dans $i$-dance : on est autour ou dedans, on peut danser, regarder, écouter. Ensuite, l'intérêt de ces objets complexes, c'est qu'il y ait de l'articulation, mais aussi de la déliaison. On ne fait pas tout en même temps : il y a des moments où on lit, où on joue, où on regarde, où on danse. Dans une exposition sur le livre, j’adorerais que l'on me laisse le temps de lire un livre en entier et, en même temps, la part du commentaire aussi est importante. Ce qui m'intéresse, ce sont ces parts, diverses, mais d'égale valeur. Que l'on soit parfois engagé, parfois non. Que l'on comprenne ou non. Une exposition ne doit pas nécessairement fonctionner de façon optimale. Car on ne sait jamais vraiment en quoi elle touche l'autre. Et ce que je défends, c'est que cet élément qui échappe fasse événement. Bien sûr, il y a des sujets qui sont plus aisés à traiter que d'autres, mais aucun n'est impossible. Je reviens de Séville où l'on me disait que les politiques demandent de faire du tourisme, pas de l'art. Or les artistes pourraient se saisir de la question du tourisme, dans une démarche artistique, décalée. Nous devons pouvoir répondre aux questions de la société, même quand elles ne semblent pas pertinentes. Pour ne pas toujours rester dans notre domaine. Cet isolement, je trouve que c'est triste. Et pour tout dire, je n'aime pas la tristesse.

\section{Propos recueillis par Julien Collonges}

\section{Notes}

\footnotetext{
1 - Pour en savoir plus sur Pierre Giner et ses travaux, on consultera son site : www.pierreginer.net

2 - Du 16 avril au 21 août 2011

3 - Stéphane Natkin, professeur titulaire de la chaire Systèmes multimédias au Conservatoire national des arts et métiers (CNAM), est le directeur et fondateur de l'Ecole nationale des jeux et médias interactifs numériques (ENJMIN). Avec Pierre Giner et Loïc Petitgirard, il est l'un des trois commissaires de l'exposition Museogames.
} 